\title{
DR-KNN: A HYBRID APPROACH FOR DIMENSIONALITY REDUCTION OF EO IMAGE DATASETS
}

Andreea Griparis, Daniela Faur and Mihai Datcu

ABSTRACT :

The two Sentinel-2 satellites provide, since March 2017, high-resolution worldwide images every five days, freely distributed, generating terabytes of high-dimensional data. An intuitive manner to summarize the main characteristics of the data and gather knowledge is visual exploratory analysis, which is often based on dimensionality reduction methods to represent high-dimensional data. From previous research and the state-of-the-art literature, turned out that t-distributed Stochastic Neighbour Embedding is one of the most appropriate technique to reduce the dimensionality of a dataset, but it requires very high computational power. To overcome this inconvenience, we proposed two hybrid DR algorithms, which combine the DR with the nearest neighbour technique or random forest regression. The main conclusion is that our approaches reduce computational power without compromising the representation quality. Index Terms - dimensionality reduction, visual exploratory analysis, remote sensing, high-dimensional data 